\title{
Automated representation of style by feature space archetypes: distinguishing spatial styles from generative rules
}

Sean Hanna

Faculty of the Built Environment, Bartlett School of Graduate Studies

University College London

1-19 Torrington Place

London WC1E 7HB

United Kingdom

s.hanna@ucl.ac.uk

\begin{abstract}
Style is a broad term that could potentially refer to any features of a work, as well as a fluid concept that is subject to change and disagreement, yet approaches to representing it too often seek either a pre-defined set of generative rules or list of measurable features. Instead, a general and flexible method of retrospectively and automatically representing style is proposed based on the idea of an archetype, to which real designs can be compared, and tested with examples of architectural plans. Unlike a fixed, symbolic representation, both the measurements of features that define a style and the selection of those features themselves can be performed by the machine, making it able to generalise a definition automatically from a set of examples. This process is implemented in analysis, and coupled with a generative algorithm to produce plans in a learned style.
\end{abstract}

\section{INTRODUCTION}

At its core, style is what distinguishes one group of works from another. This paper is concerned with representing styles, particularly in a way that is able to capture the way they can evolve and change over time, and with automating this process, both as a means to understand it and as a potentially useful tool in design. It proposes that we can define a style using an archetype, an ideal model comprised of the features that exemplify the style. This concept differs from the description of a type, or category into which particular examples can fall, and from that of a prototype, precedent or case, which are actual instances on which later examples can be modelled. An archetype is something between the two, a generalisation that cannot exist materially, yet matches and is compared to many actual instances. This is almost certainly not a real example, but an abstraction made up of only those features necessary to differentiate it from other archetypes. 
If one imagines a particular style, that of Palladio for example, certain features such as axial symmetry, classical orders, etc. come to mind immediately because they are common to that style. Others, like the colour of the roof tiles perhaps do not, either because they are not shared (the Villa Rotunda is red, San Giorgio Maggiore is white), or because they are ubiquitous (the majority of Italian roofs have always been terra cotta). Yet even this set of features can not be predetermined, as it may differ from style to style. In human terms, it is this set of essential features that constitute an archetype. This paper proposes a computational and algorithmic equivalent.

For the production, as opposed to the analysis of style, many approaches utilise a generative rule system, by which examples of the style may be built. Just as a generative grammar [1] is capable of producing all well formed expressions of a language, these rule systems are capable of producing examples of designs. As an approach to representing style, a given rule set is often thought to encode the style [2-4], such that it produces designs that are examples of it, and can therefore serve to represent it. This is a useful analytical and pedagogical abstraction, but gives limited insight into how we actually design, transmit or communicate styles, or how they can change. Linguistic grammars themselves are simply formal methods, and not thought to represent the actual cognitive processes involved in understanding or producing language.

In language, however, it is not the grammar that defines style, but specific choices within it - the same English grammar, for instance, is capable of producing romantic, utopian, realist, symbolic or an innumerable number of different styles of literature. Similarly, the present work separates the style definition from the generative rules themselves, by allowing the archetype to guide such choices within the rule system.

This archetype is based on the mapping of design examples in a high dimensional feature space, and uses methods of dimensionality reduction of this space to yield an archetype that describes the style. This can be used to classify, and as a measure to generate new designs. The use of a feature space agrees with our own intuitive ability to evaluate designs as being stylistically nearer or farther from one another, and is commonly applied in machine learning. The nearest neighbour algorithm [5], for instance, classifies an unknown example of data by simply measuring its distance to previously known and labelled examples, or prototypes. 
Two innovations are proposed over such existing methods. The first is that the archetype is a generalisation that combines both the concept of the ideal example and the particular space in which it is measured, consisting only of the features relevant to that particular style: it is comprised of both a mapping to a feature space and a point within that space. The second innovation concerns the synthesis of new designs, and incorporates the notion of affordances [6], to consider the design process as a continual evaluation and choice between possible alternatives as the development of the design progresses. These choices can be made by repeated measurement against the ideal that the archetype represents. Figure 1 presents a schematic diagram of the method to be described in sections 4 and 5.

The approach was tested within the design domain of architecture, using the plan in particular, and the two major aspects are implemented in sections 3 and 4. The first deals with analysis, and begins by providing methods by which plans of real buildings can be embedded in a feature space such that those that are similar fall near to one another. This yields a way in which all styles might be understood and represented by a computer, which is not based on any predefined symbolic representation. The second part refines this spatial embedding, and combines a very simple generative process to synthesise designs.

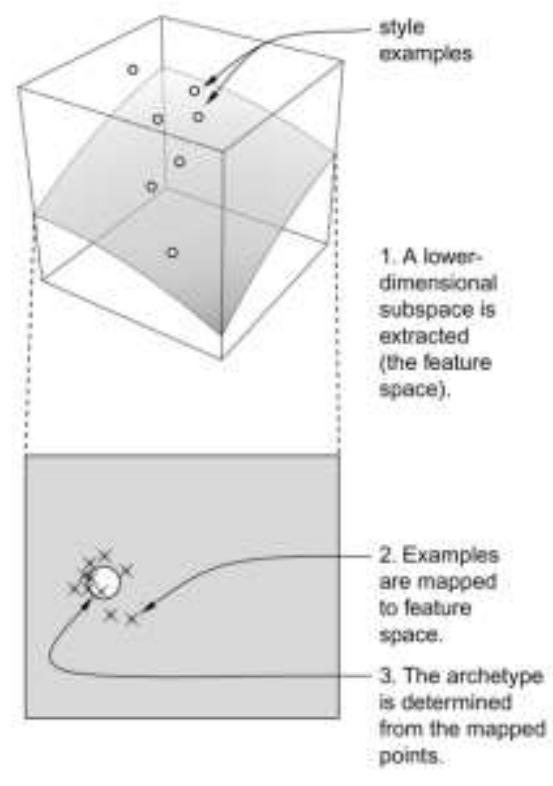

a) Defining the Archetype

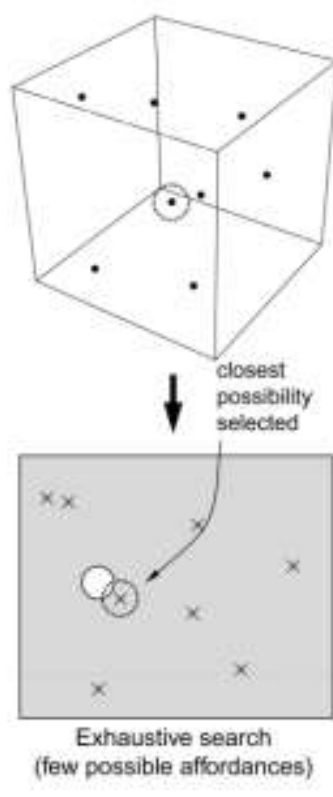

b) Generating New Designs
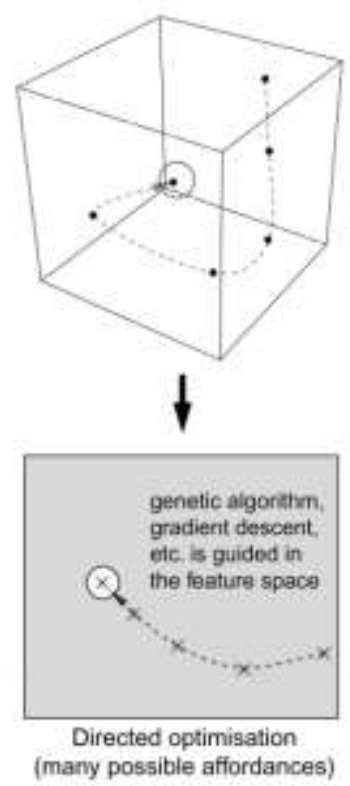

(many possible affordances)

Figure 1: Diagram of the method of (a) deriving the feature space and archetype from examples, and (b) generating new designs by selecting affordances in the feature space. 


\section{APPROACHES TO ARCHITECTURAL STYLE}

The need to differentiate is fundamental to perception. Gombrich [7] suggests that art provides categories by which to sort our impressions: 'without some starting point, some initial schema, we could never get hold of the flux of experience.' Like a game of 'Twenty Questions', where the actual identity of a concept is gradually communicated through trial and error, the symbols of art do not represent reality in all its detail, but only what is necessary to convey the message. In his view the set of symbols used constitute the style, and approaches to representing style, in general, are rooted in identifying the equivalent of these symbols, either as features or generative rules of the work. Architectural style has been represented a number of ways, both for analysis of existing designs, and for synthesis of new ones.

\subsection{Generative approaches}

Rule systems have also been developed to generate new designs in a particular style, such as Hersey and Freedman's computer implementation to create possible Palladian villas [2]. Shape grammars [8,9] are possibly the most widely studied, and have been successful in providing generative descriptions of many styles. A descendant of linguistic generative grammar [1], they provide an explicit rule-based method for producing final designs, and have yielded examples in the apparent styles of Palladian villas [4] and Frank Lloyd Wright's prairie houses [3]. Recent approaches have expanded the method to allow decompositions on finished or existing designs to generate new rules for design exploration [10].

As an approach to style, the style is often (e.g. the examples above) encoded with a specific grammar. A creative human then works with this grammar to make a specific design within the style. As a tool for analysis, the grammar or rule set is constructed by a human designer, a fully automatic process seen as undesirable or impossible [11]. In its generative capacity it is then followed by a user choosing which rule to apply at each stage in the process to create designs comparable to originals.

\subsection{Analytical approaches}

Analytical methods have been proposed that model similarity or group designs based on a count of predefined features. Chan [12] uses a list of material and compositional features to evaluate buildings as to their style, and finds a correlation with the judgements of test subjects. Experiential qualities of architecture have also been structured or mapped to rule sets to guide architects [13-15], and this approach has been 
implemented by Koile [14] in an expert system that is also able to recognise style as a count of defined design characteristics.

Another approach quantifies style by various measurements taken from the works. More general analytical techniques using information theoretic measures have been used to measure distance between individual plans [16], and to derive values for measurements such as entropy [17], morphology and topology [18] that can be used to evaluate examples of a style. These have the advantage of quantifying designs of any style as real values on the same scales, so that variations within or between styles can be measured uniformly.

\subsection{Defining style retrospectively}

The goal of naming a particular class of measures that we can specify in advance to contain the description of all styles is misguided. While the choice, a priori, of explicit rules or feature definitions can tell us interesting things about a style, they are often a simplification that will either produce some designs that would be considered outside the style or fail to produce all possibilities within it ([2], ch. 2). Style is often considered (as in [7]) the method of expression as opposed to the content, but Goodman [19] argues for a broader definition of style to include aspects of both what is expressed in addition to how. In his definition, the style 'consists of those features of the symbolic functioning of a work that are characteristic of author, period, place or school', and these can only be determined in relation to the works, not beforehand.

In deriving a method for determining and measuring these features then, several properties evident in real design processes are desirable:

- A method should exist for deriving style from examples: In designing, we often cannot state a goal explicitly, only point to examples. Styles are only discernable after several similar designs exist, and it is through the emulation of examples, not by recipe, that a style is communicated.

- Styles are not clearly bounded, and are subject to evolution and change: Even the seemingly clearly defined classical orders, before being codified by Vitruvius [20], were the result of centuries of experiment and still subject to debate on particular proportions throughout the Renaissance. 
- Styles are divisible to ever finer distinctions: There is a continuum of stylistic differences (Gombrich's 'flux of experience'), in which a style may be distinguished by period, movement, designer, but only down to the level of the individual work.

The method proposed here is intended to reflect these properties of style.

\subsection{Generating designs}

A simulation of the design process in all its detail may be impossible, but complexity studies indicate that in modelling such systems it is the basic structure of interaction that is required [21]. Ideally this is reflected in the generative method, as in the choice of a bottom up aggregation rather than a top down division in cities that are built over time $[22,23]$.

To allow flexibility inherent in the properties above (Section 2.3) there are two possible structures through which the generative method and style representation can interact:

- Style is represented in a generative rule set: the rule set must be constantly re-created, allowing it to evolve. The possibility has been suggested [10] that new grammatical representations, for example, can be created at any step in a continuing design process.

- Style is determined by analysis: a single rule set is capable of generating many different styles/types, each of which is defined not by the rules but the choices made within it.

This paper takes the position that the second forms the greater part of the intuitive process we know as design and shows how it might be implemented in a computational model. It differs from previous approaches in that design examples will be evaluated by a general analysis, then the relevant features determined automatically in the definition of the archetype. By so doing, both the processes of defining a style and building examples of it can be performed by a machine.

\section{RELATED TECHNIQUES}

Several techniques from related fields of machine vision and space syntax are relevant to this work, and are outlined here before their use in later sections.

\subsection{Feature description by dimensionality reduction in other fields}

The automatic generalisation of a description of style from examples has occasionally been explored in other domains such as musical style [24,25], but is more firmly established in techniques of machine 
classification and learning used in fields like machine vision. Dimensionality reduction is often used in applications from face recognition [26] to linguistic analysis [27] to infer distinguishing features from a given set of high-dimensional data. Principal component analysis (PCA) provides a new set of axes aligned to the characteristic vectors, or eigenvectors of the covariance matrix of the data set. The dimensions in which the data varies least are discarded to yield a lower dimensional subspace of the data that can then be used to make classifications.

The principal components of face images, for example, referred to as 'eigenfaces' (Figure 2) [26], are used by face recognition software to effectively quantify a new face by how it measures against each, and its best match found from existing data. More closely related to our experience of architecture is the problem of a robot visually navigating through a real, unstructured environment. Dimensionality reduction has been used on the image data recorded by the moving camera of a robot's visual system to allow the computer to generalise its own concepts for objects, which generally correspond to objects such as 'tree' or 'rock' [28]. It makes the same kinds of distinctions and classifications that we would, thereby allowing it to navigate.

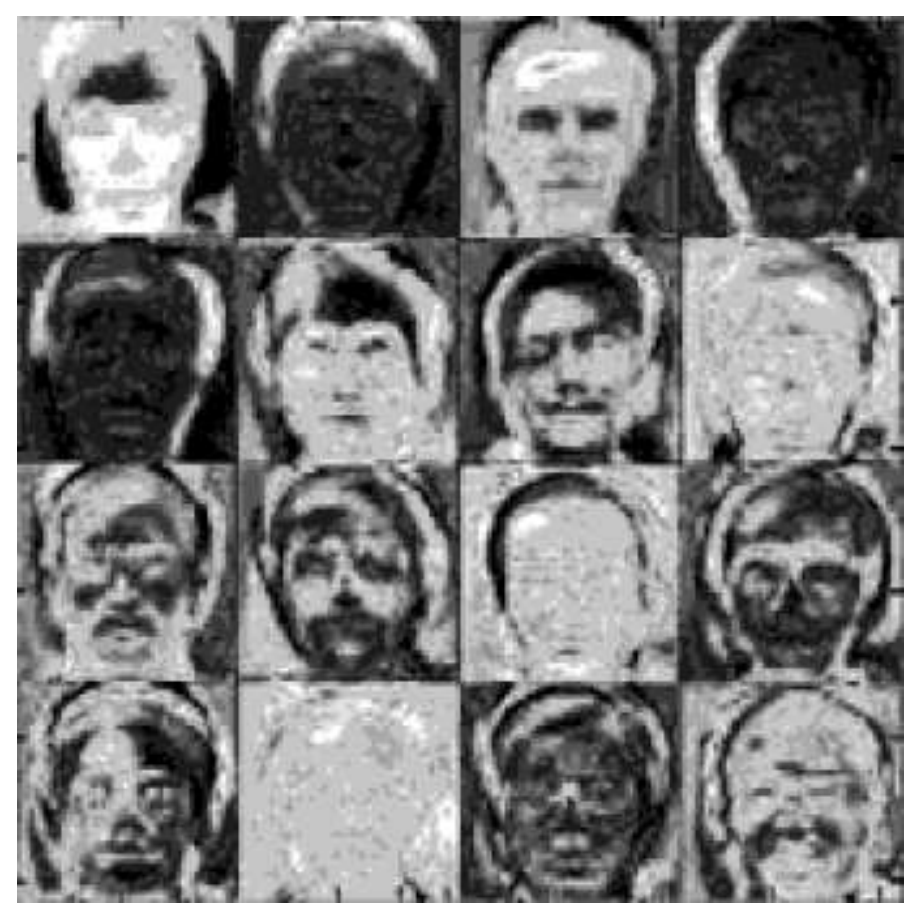

Figure 2: 'Eigenfaces': the first sixteen principal components of a data set of face images. 


\subsection{Representation of spatial features as graphs}

To represent stylistic features of space, the computer requires an appropriate substitute for the sense data provided by the images or sound digitisations in the applications above - a method through which to perceive experiential qualities of the building that is as generic as possible. Two related space syntax techniques: visibility graph analysis [29] and axial line analysis [22,30] both provide an approximation of how people actually experience a space using the unobstructed sightlines of the plan, represented as a graph. This is not to suggest that these graphs necessarily capture all features that all our human senses may determine to be relevant to a style (certainly materials and details are not represented), but that within a certain scale they constitute a reasonable generic experience for the computer.

Properties of visibility and axial graphs have been shown to be strongly related to both spatial perception and resulting behaviour of people within spaces. Strong correlations have been found with measures of visibility graphs and observed way-finding, movement and use in buildings [29], and urban pedestrian movement [31]. Axial graphs have likewise been shown to be closely related to directly observed movement [32,33], building use and social interaction [34], and indirect behaviour such as land values and crime $[35,36]$.

\subsection{Measuring between graphs}

Several approaches to similarity measurement have been based on small graphs of adjacency or connectivity of spaces in plan. String edit distance [37] and measures of similarity or complexity [16] have been used effectively for small semantic or adjacency graphs. With larger and more complex graphs as generated by axial lines, calculation of similarity becomes more difficult, but this can be overcome with graph spectral analysis, a technique shown effective in image recognition [38]. Spectral analysis of a graph uses the eigenvalues and eigenvectors of its connectivity matrix, and is relevant to the kinds of analysis considered here. The leading eigenvector, for instance, gives the result of a steady state random walk on the graph, equivalent to the connectivity of sightlines as described above. The spectrum of a graph, or ordered set of eigenvalues, is useful in that it can be used to represent the graph as a single feature vector.

\section{ANALYSIS: REPRESENTATION OF A STYLE IN FEATURE SPACE}


The archetype feature space must be derived initially from a general input capable of containing features relevant to all styles. This section tests both the acceptability of the axial graph as sense input, and the general use of a feature space for real building plans.

It is proposed that the description of a style is inherent in the examples of that style, and so examples of one style are objectively more similar to one another than to examples of other styles. The stylistic description should therefore not be needed a priori to make this distinction, but the algorithm should be able to make the same kinds of classifications that we would, without explicit training. In this section a feature space is found for a group of buildings by unsupervised dimensionality reduction as in the examples in section 2.2. The examples are found to naturally fall into recognisable clusters within the space without the need for explicit labelling, and these correspond to the buildings' inherent similarities. This will provide the basis from which to derive an archetype from the examples.

\subsection{Description of plan styles in a feature space}

An initial test to describe styles in a feature space used a set of 24 plans, taken from various twentieth century iconic buildings [39]. This involved a simple dimensionality reduction of a feature space to confirm the hypothesis that proximity does indeed indicate stylistic similarity.

Axial graphs were constructed for each of the 24 samples, and this data - in effect a set of binary adjacency matrices - was taken as the computer's raw sense data, or experience of each of the spaces. Analysis was performed using Depthmap software, which constructed a minimal axial graph based entirely on plan input and an objective algorithm [40]. Figure 3 displays the lines of sight in Frank Lloyd Wright's Fallingwater shaded to indicate the degree of spatial integration. Darker lines clearly reveal the higher traffic zones that link the rooms of the house. The spectrum of this graph directly captures connections between these spaces and was taken to form the vector from which relevant features would be derived. 

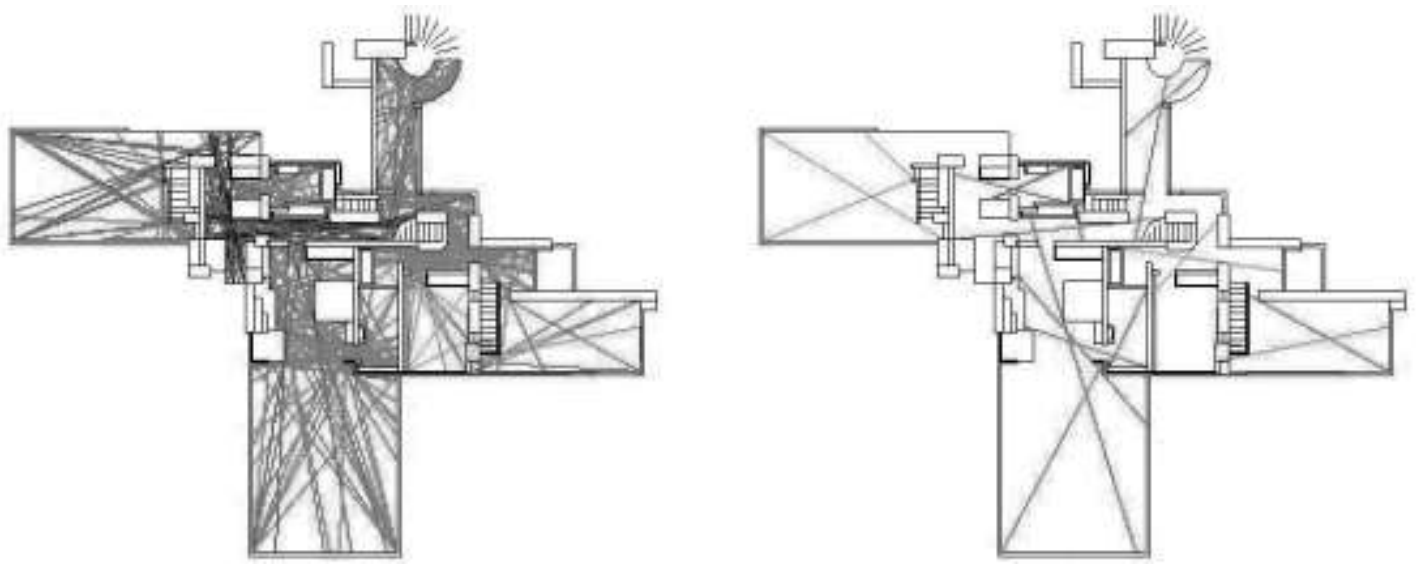

Figure 3. Left: A complete axial graph of Frank Lloyd Wright's Fallingwater. Right: The reduced minimal graph.

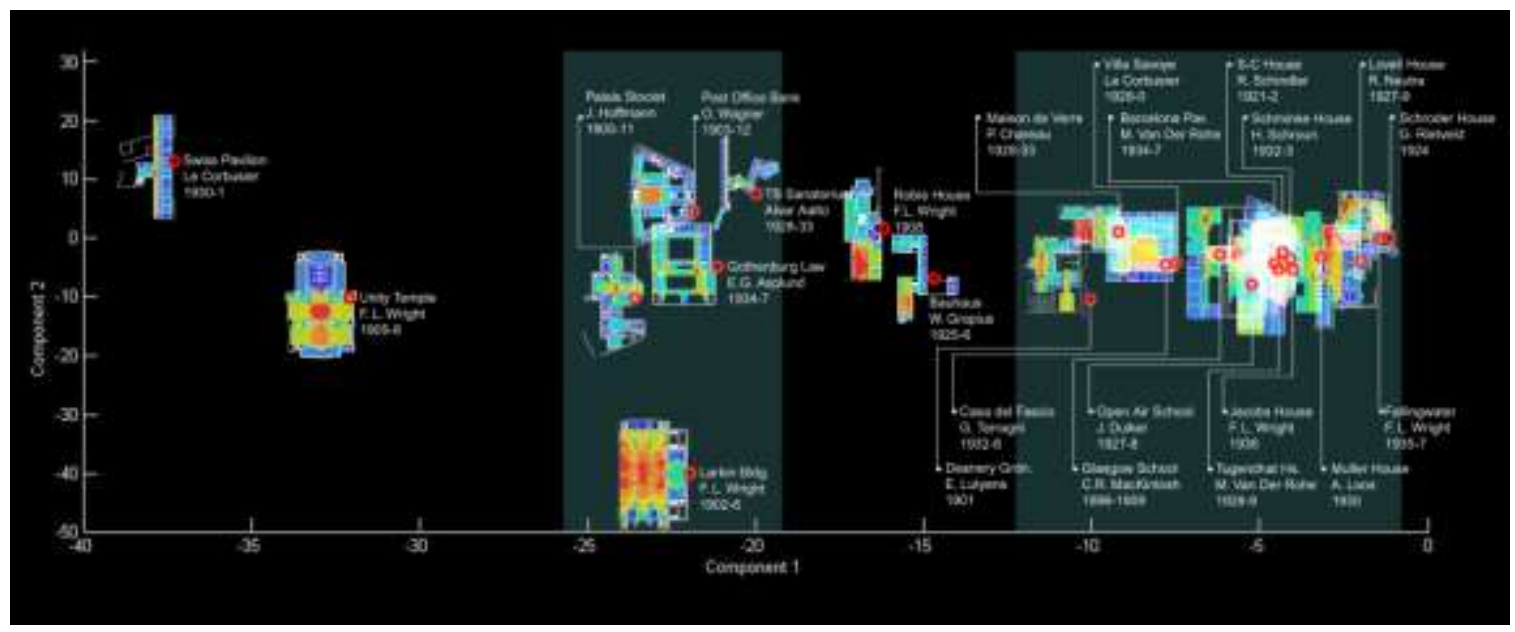

Figure 4. The example buildings plotted in feature space.

With the spatial analysis of each plan quantified as a vector, the example buildings can then be positioned in a high dimensional feature space, with each value in the spectrum on a different dimensional axis. PCA determines, for a given set of data, the combination of dimensions in which it is most likely to vary, and these are used as new axes in a reduced version of the space that captures the essential statistical features of the data set. A reduction of the plans' feature space based on the first two principal components of the set is shown in Figure 4. The dimensions of this new feature space are strictly computational, and are meaningful only in a statistical sense, rather than in the sense that they could be easily described. The first component, 
on the horizontal axis, represents a combination of the features in which the plans are judged by the algorithm to differ the most.

Yet it can be seen that these include meaningful features such as typology (most houses toward the right and larger, public buildings to the left) as well as general stylistic trends. The shaded groups indicate the proximity of most of the axially symmetrical, pre-Modernist buildings to one another, as well as rough zones of early, and of high modernist buildings, typically with spaces that flow into one another articulated by shifting orthogonal planes. There is an approximate chronological order from left to right, seen clearly in the buildings by Wright, the Villa Savoye and contemporary Maison de Verre are next to one another, and van der Rohe's two works are virtually overlapping.

The divisions in the diagram are drawn simply for clarity, and are not meant to suggest a division into distinct groups. The points as plotted at this point represent a continuum in a uniform space. It is true that van der Rohe, Le Corbusier and Wright can be considered to have quite different styles, as can different buildings by Wright alone, but proximity in this feature space is meant to suggest those buildings that are more similar to one another by degrees. The only significant outliers in this regard seem to be those caused by typology: the private houses vs. the public buildings, but at this point no attempt has been made to draw the distinction. (The machine learning algorithms to be described in section 5 will allow this.) The fact that buildings of similar styles do fall near to one another in the reduced feature space confirms that the features indicated by the principal component are at least statistically related to style. This suggests that archetypes based on such a space may be used as style descriptors.

\subsection{Classification of plans into distinct sets}

The plans above form a sort of continuum as they are taken arbitrarily from various styles over a forty year period, but the same process of graph analysis and dimensionality reduction can be used to plot the features of a particular group, and thereby define it. The above method was used again on a more focussed set of only two contrasting types in which there could be no ambiguity as to the label of each. A set of 40 sample plans (Figure 5) was used containing examples of two building types: modern offices, and neoclassical museums. The experiment tested the degree to which the machine classification corresponds to our own, human decisions, and so these groups were specifically selected by the author as sets that can be distinguished by a human judge. Distinct building types were used, rather than simply contrasting styles, to 
avoid any ambiguity (as in section 4.1) as to the distinction of style at this point, and avoid as much as possible the experimenter's bias. Plans were chosen such that in overall shape the two groups are similar to one another, ensuring that the classification is based on more subtle details.
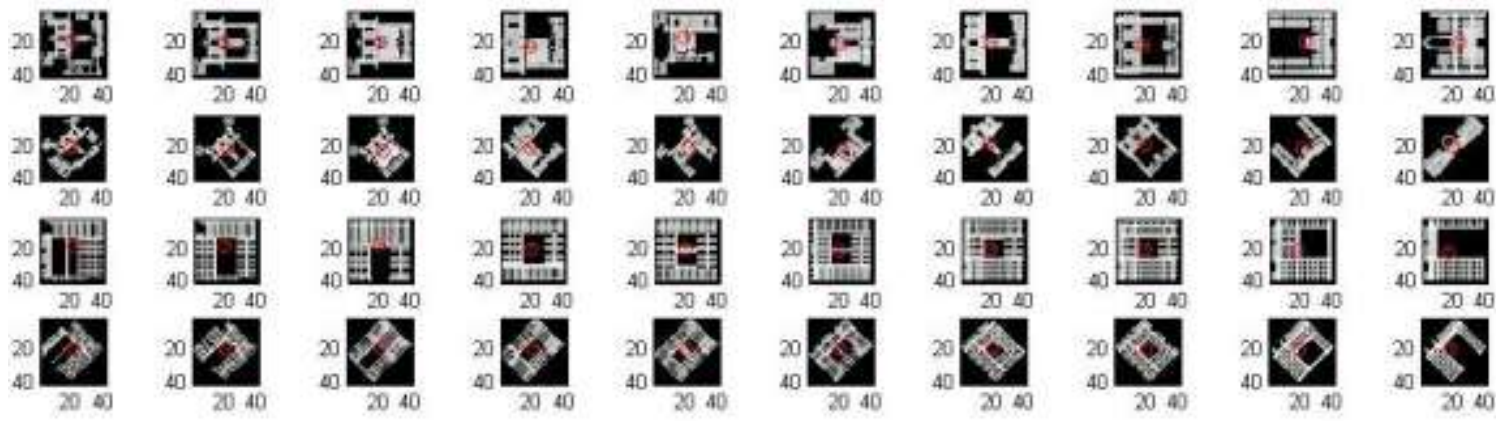

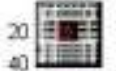
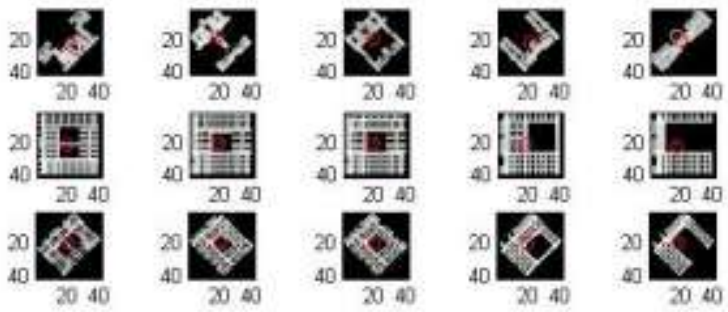

Figure 5: The upper 20 plans representing museums, and the lower 20 offices.
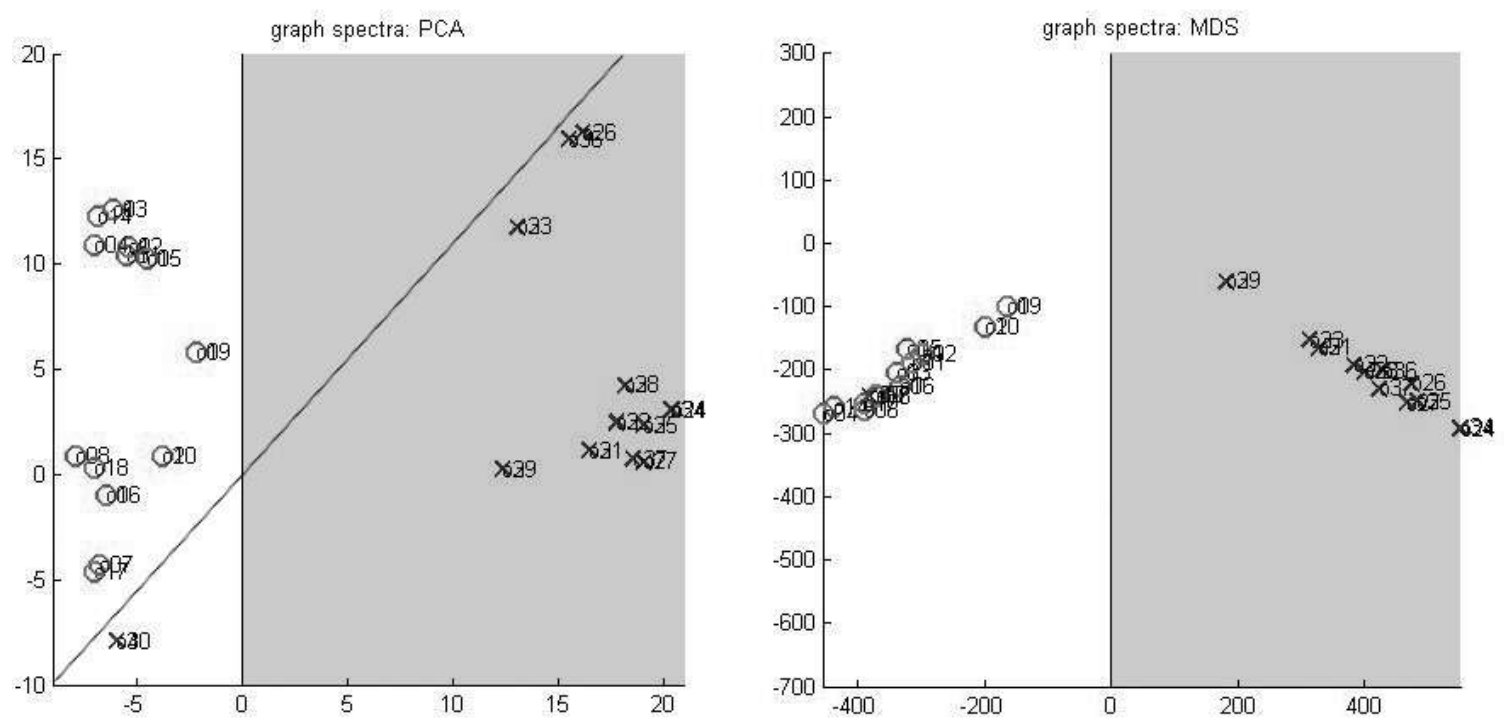

Figure 6. Left: The plans are classified by the machine by PCA. Right: By multi-dimensional scaling. The horizontal axis is the principal axis of the data set; the vertical axis is the second.

In Figure 6 (left), PCA is performed on the spectra derived from the axial graphs, and the results plotted against the first two components (again, derived statistically from the data). It can be seen that the two groups of plans can be almost entirely classified even on only the first principal component (horizontal 
axis). There is a clear separation into two clusters with a large margin between. The museums, marked by ' $O$ 's, are distributed over a region to the left of the origin, and the offices, marked by blue ' $\times$ 's, are toward the right. An outlier from the office group can be accurately classified as well using another dimension of the second principal component. A multi dimensional scaling method (Figure 6, right) was also used as an alternative to PCA, resulting in an even clearer distinction.

No supervised training was required at this point. It simply indicates that in all the most obvious ways (obvious to the unsupervised computer) that the plans differ from one another overall, the plans within each type are more similar to one another than to those of another type, and that this distinction can be made by the algorithm.

\subsection{Defining archetypes by the clusters}

The setting of an archetype from such a clearly classified plot requires only the selection of the point that best describes the region of reduced feature space in which the examples of that style lie. Nearestneighbour algorithms for analysis define prototypes as the generating points of the Voronoi tessellation that separates the classes (Figure 7, left) [5], but for the synthesis to follow in section 4 this would bias the result when these lie close to the edge of a cell. Also, for only two classes there are infinitely many such points. The point that minimises the distance to all examples in the cluster is simply the mean,

$$
\boldsymbol{\mu}=\sum_{i=1: \mathrm{n}} \varphi\left(\mathbf{x}_{i}\right) / n \quad \forall \mathbf{x} \in \mathrm{S},
$$

which can be applied as easily for two style clusters or two thousand. This mean and the mapping to the reduced feature space

$$
\left\{\mathrm{z}_{1}, \ldots, \mathrm{z}_{j}\right\}=\varphi\left(\left\{\mathrm{x}_{1}, \mathrm{x}_{2}, \ldots, \mathrm{x}_{i}\right\}\right),
$$

where $j<i$, together constitute the archetype (Figure 7, right).

The lower dimensional feature space that results allows a description of style that is convenient for analysis and measurement - in that any plan example can be evaluated, and compact - in that only a few dimensions need be used. Because most of the dimensions have been removed the space itself comprises 
only those features that are relevant to differentiate examples of one style from another, and the mean point of each of the clusters above can be said to be the archetypal ideal of each group. Like any archetype it is a generalisation, and examples that fit it exactly may not ever be found in the real world, but it can serve as a model to guide the creation of new designs.
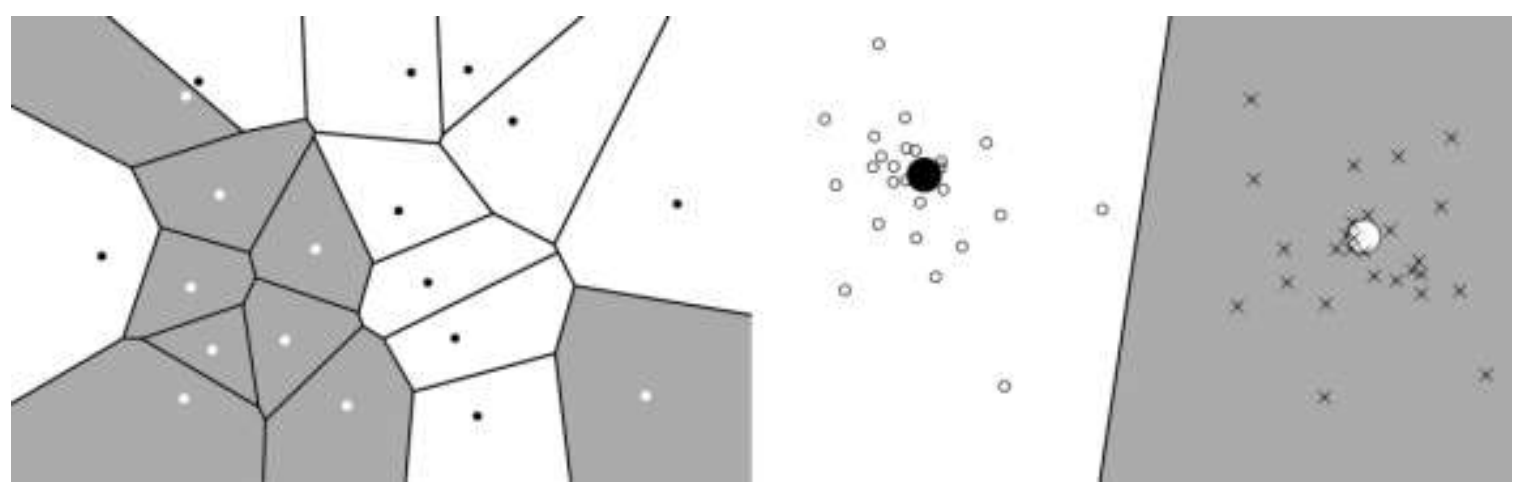

Figure 7. Left: Nearest neighbour prototype points define Voronoi regions of the original feature space.

Right: Archetype points $\boldsymbol{\mu}$ at the mean of the examples define regions of the reduced feature space.

\section{SYNTHESIS: PRODUCTION OF NEW DESIGNS}

In this section more sophisticated classification algorithms are used in place of PCA to derive the features, and methods for improving stylistic fidelity will be investigated. The use of supervised learning with labelled examples will imply a reduced feature space that is not just statistical, but meaningful. Analysis will also be combined with a generative method to synthesize new designs, and these will be used to evaluate the success of the archetype representation.

This furthers a stream of research begun to explore the transmission of building styles between collaborating individuals [41]. Some of the previous results are shown again in this section, but in the context of representing stylistic concepts for design synthesis by individuals rather than the context of group creativity and communication.

\subsection{Design as selection of affordances}

Style can be considered a choice between given alternatives [7], but rather than seeing this as a choice between generative rule systems, it can also be a choice of design moves within a system. While it may not 
be conscious [19] the act of creation implies the repeated choice of one design decision over another. One might consider this the ongoing selection of what Gibson [6] terms affordances: opportunities for action provided by the environment, or in this case the design, as the design evolves over time. At any stage of the design of a building there are only certain possibilities open to the architect, and the act of adopting one style over another can be seen in this sense as a selection from the afforded alternatives. Tang and Gero [42] suggest the act of sketching, with constant drawing and re-evaluation is such a process, choice of rules is the inherent design activity of shape grammars, and the explicit representation of choices as design spaces has also been proposed for CAD environments [43]. In this section a generic system will be used to generate designs, but the style will be expressed by the choices made within it.

\subsection{A basic open generative system: building aggregation}

No generative algorithm is a direct counterpart to the axial graphs used in section 3 , but there is one that precedes them. Axial analysis was developed initially to analyse the variation in settlement layouts, particularly in French and English stylistic variants of what was termed the 'beady ring' form [22]. This form itself was found to be the natural result of a very simple but open generative system of aggregation, the basis of which will be used to produce new design examples in this section.

The grid-based model allows pairs of open and filled cells to aggregate randomly, with the restriction that each new pair must join its open cell to at least one other open cell already placed, and the closed cell does not join another closed cell only at the vertex. Each time the model is run, the emergent global structure forms that of the beady ring settlements studied, with a chain of open spaces onto which inner and outer groups of buildings face.

More important for the question of style as differentiation are the specific differences between towns: French vs. English, for example. Although these were stated to be the result of variations in the application of the generative rules ([22], pp. 84-85) the model was not extended to make anything but random choices. In this section, the decisions of aggregation will be made instead by the evaluation of each possibility against an ideal specified by the archetype, and the choice of that possibility which fits best.

Although initially applied to town formation, this aggregation model is sufficiently general to represent rooms and corridors in a building or desks and chairs in an open plan office, depending on scale. While it uses a simple and constrained morphology, the grid is still able to represent configurations of very 
different patterns by the choices made in aggregation, and so it can stand as an analogy to more sophisticated generative methods to demonstrate the principles of this paper.

\subsection{Two styles of aggregation as examples}

Two artificial stylistic norms were chosen to be easily distinguishable from one another, and a simple algorithm written to aggregate open/closed pairs of units in the manner of each. The first is a strict arrangement of straight rows rather like highly planned settlements such as Manhattan, and the second is a random arrangement of units joined open cell to open cell (Figure 8).

While the perception of spatial qualities of existing building plan examples in section 3 required the construction of axial graph matrices, in this simplified grid model samples were taken directly. Each time a new pair is placed in the plan, its relationship to the $7 \times 7$ cell neighbourhood surrounding the open half of the doublet is taken as an example $\mathbf{x}$. The 49 cells, each containing either a closed building (indicated by a filled square or 1), a public open space (a dot or -1) or yet unbuilt (empty or 0) are used as the computer's sensory experience of that particular built example.
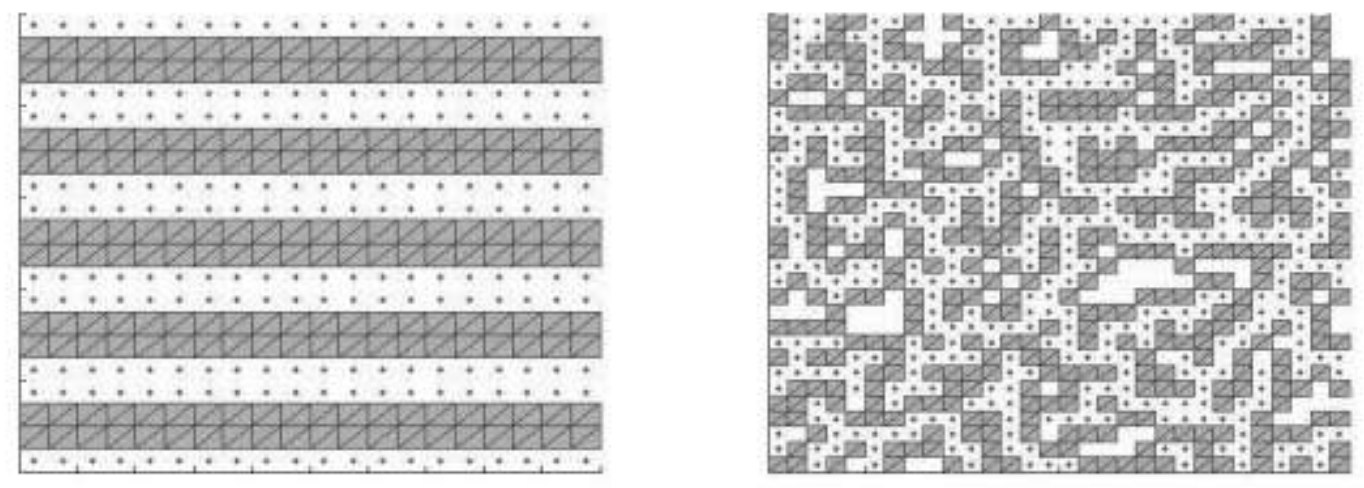

Figure 8: Two styles: strict rows and random aggregation. 


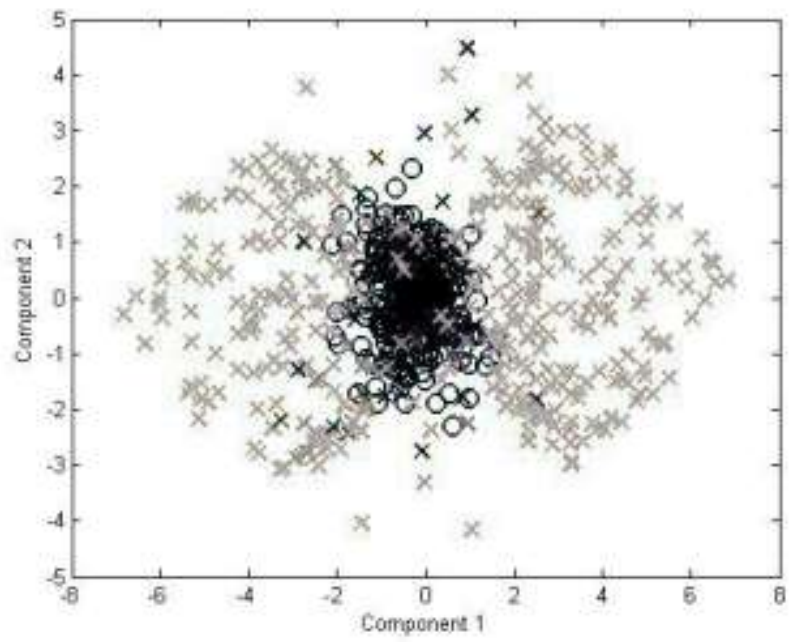

Figure 9: Examples from a 49-dimensional feature space.

As in the case of the plan graphs, these neighbourhoods are points in an initial feature space. Each unique example can be represented by a point in a 49-dimensional space, a 2-dimensional projection (by principal components) of which is shown in Figure 9. Neighbourhoods of the straight rows are indicated by ' $\times$ ', and the random style by ' $\circ$ ' markers in the centre.

\subsection{Learning and building to an archetype}

Because clear clusters are less evident than in section 3, a supervised learning algorithm is used to provide the mapping $\varphi(\cdot)$ in eqn (2). In the resulting space, the mean $\boldsymbol{\mu}$ of the cluster represents an archetype of that style to be used in a straightforward building algorithm: at every step a given number of positions and orientations are available to be built, and the decision is simply the act of choosing which one of these affordances is closest to this ideal:

$$
\min (\|\varphi(\mathbf{x})-\boldsymbol{\mu}\|) .
$$

Three experiments test the method of learning an archetype from examples and building to that archetype. The first tests that the style can be learned, with the hypothesis that clearer clustering will lead to a better resulting generation of the style. The second reveals that results can be improved by using a unique 
feature space reduction for each archetype. The third tests the hypothesis that the results of construction are independent of the choice of learning algorithm and particular representation of the archetype.

\section{Clustering in a feature space and clarity of style}

The hypothesis that learning enables styles to be produced implies a correlation between the degree of clustering in the feature space and the strength of style in the resulting design. A support vector machine (SVM) [44] was used to test this by adjusting the former with a tuneable parameter. SVMs operate by finding a maximally separating hyperplane between the two labelled classes in a higher dimensional representation of the input, and that representation is given by a non-linear function with a parameter that can be used to adjust the fit to the data - in this case the width of a Gaussian.

Figure 10 plots the SVM output for increasing values of $\sigma^{2}$ at left, and the effectiveness of the classification is indicated in the second column images by the shading of each sample. It is evident from the results that as $\sigma 2$ increases, the cleaner separation between the two groups by the algorithm results in a clearer construction, as shown in the images to the right. At each construction step, the possible sites and orientations are evaluated by the SVM, and the one closest the mean of either style as learned is selected. The completed constructions over a period of time are shown, one emulating the rows style as learned and the other the random arrangement, and the overall patterns are most easily seen for $\sigma^{2}=25$, particularly for the straight rows.

The initial hypothesis is confirmed, but the separations in Figure 10 are never quite enough, and the classifier can only produce adequate rows with an artificially created set of 'perfect' examples of row neighbourhoods. These are all identical, so that each is exactly perceived as the ideal archetype, and consequently the perfect classification of the two groups results in a stronger expression of the style.

(Figure 11.) 

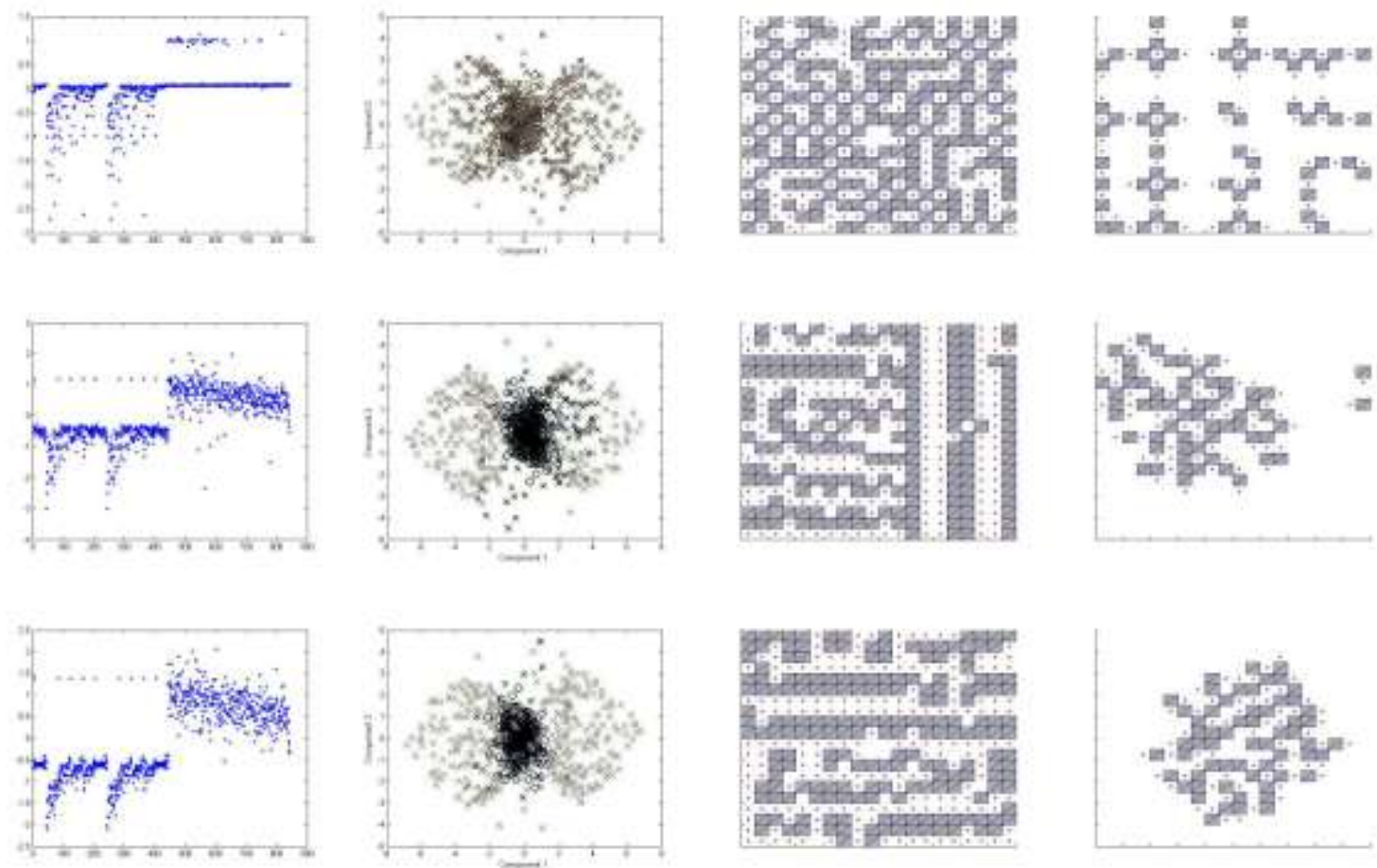

Figure 10: Building results for algorithms trained with a SVM: $\sigma^{2}=5$ (top), 15 (centre) and 25 (bottom).

The first image on the left shows the mapping of 900 examples against the vertical axis. The second indicates apparent membership in each cluster by the shading of the points. Resulting building patterns follow emulating rows, then random aggregation.
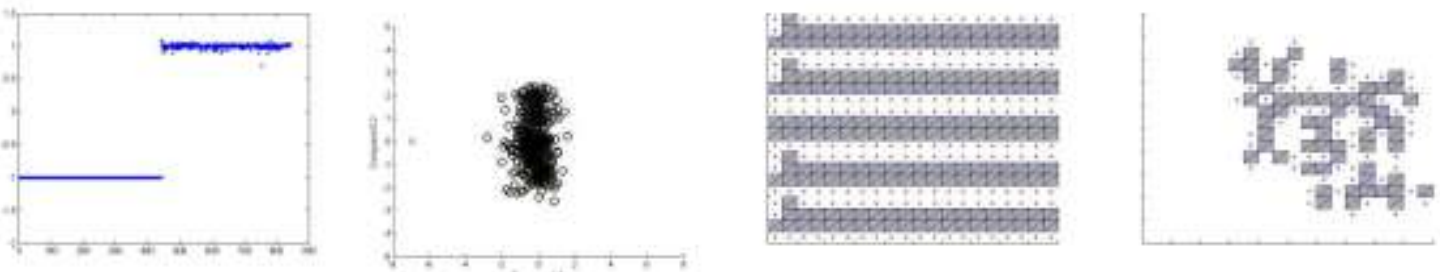

Figure 11: The same training on a set of 'ideal' examples.

\section{Clarifying the archetype feature space: training by neural network}

Features can not be chosen a priori because styles do not all differ in the same features. Rather than using a single feature space for classification, this experiment attempted to produce stronger results by finding a 
unique feature space fit to a single style, in which the points are clustered closely together as differentiated from all others.

A neural network was used to learn the rows style only, with the random examples serving as mere background from which to differentiate the relevant features. A Feedforward Multilayer Perceptron (MLP) [45] was used, with 49 input nodes corresponding to the state of the neighbourhood, 50 nodes in the hidden layer, and a single, linear output that rates each example. Training was conducted by exposing the network to 450 examples from each of the two test styles and backpropagation of errors. The examples outside the style should require no target, so a modified error function was used:

$$
J_{k}= \begin{cases}1 / 2\left\|\mathbf{z}_{k}\right\|^{2} & \text { if } k \in \mathrm{S} \\ 1 / 2\left\|1 / \mathbf{z}_{k}\right\|^{2} & \text { if } k \notin \mathrm{S},\end{cases}
$$

where $\mathrm{S}$ is the set of examples of the style to be learned (rows).

Results of this style-specific feature space were superior to those of the SVM in section 4.4.1., with most of the examples in $S$ (the row units) appearing at 0 in the feature space, and most of the others as far away (note the extreme scale of the output axis). The resulting aggregation of open and closed cells produced by the building algorithm very closely resembles that of the original rows from which it was trained (Figure 12).
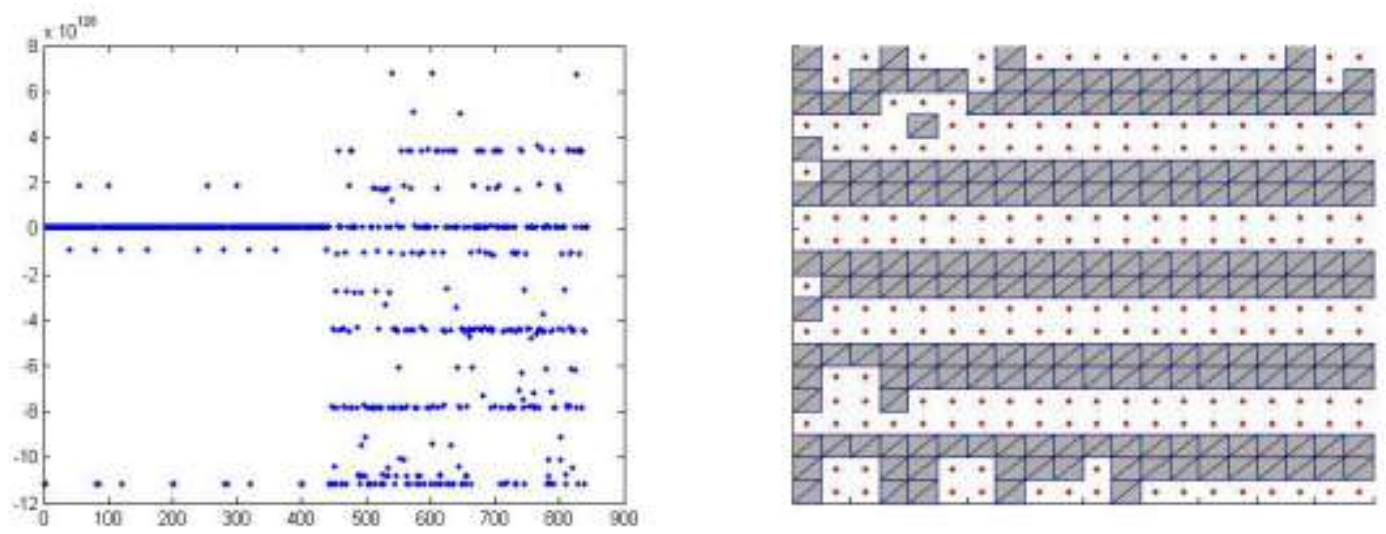

Figure 12: Training of a three layer network on the row samples. 


\section{Variations on the representation to learn the same style}

Because the style is described by a feature space rather than symbolically, the actual method of feature space mapping in the archetype is quite arbitrary. The third experiment tested that it can be changed and still lead to recognisable output. Figure 13 shows the result of several very different learning algorithms exposed to the same set of examples, each resulting in a very different mapping of features (left) but very similar overall construction of rows.

Interestingly, like Gombrich's game of 'Twenty Questions' in which the initial questions can also be arbitrary, the choice of classification algorithm used to define the style does not appear to matter. The similarity of the final constructions indicates a style can be represented many different ways. Even with the constrained grid morphology of design space, there is a drastic difference in the feature spaces (Figure 13, left). The only necessary common process is that each forms an archetype in its unique feature space based on the examples of that group. 

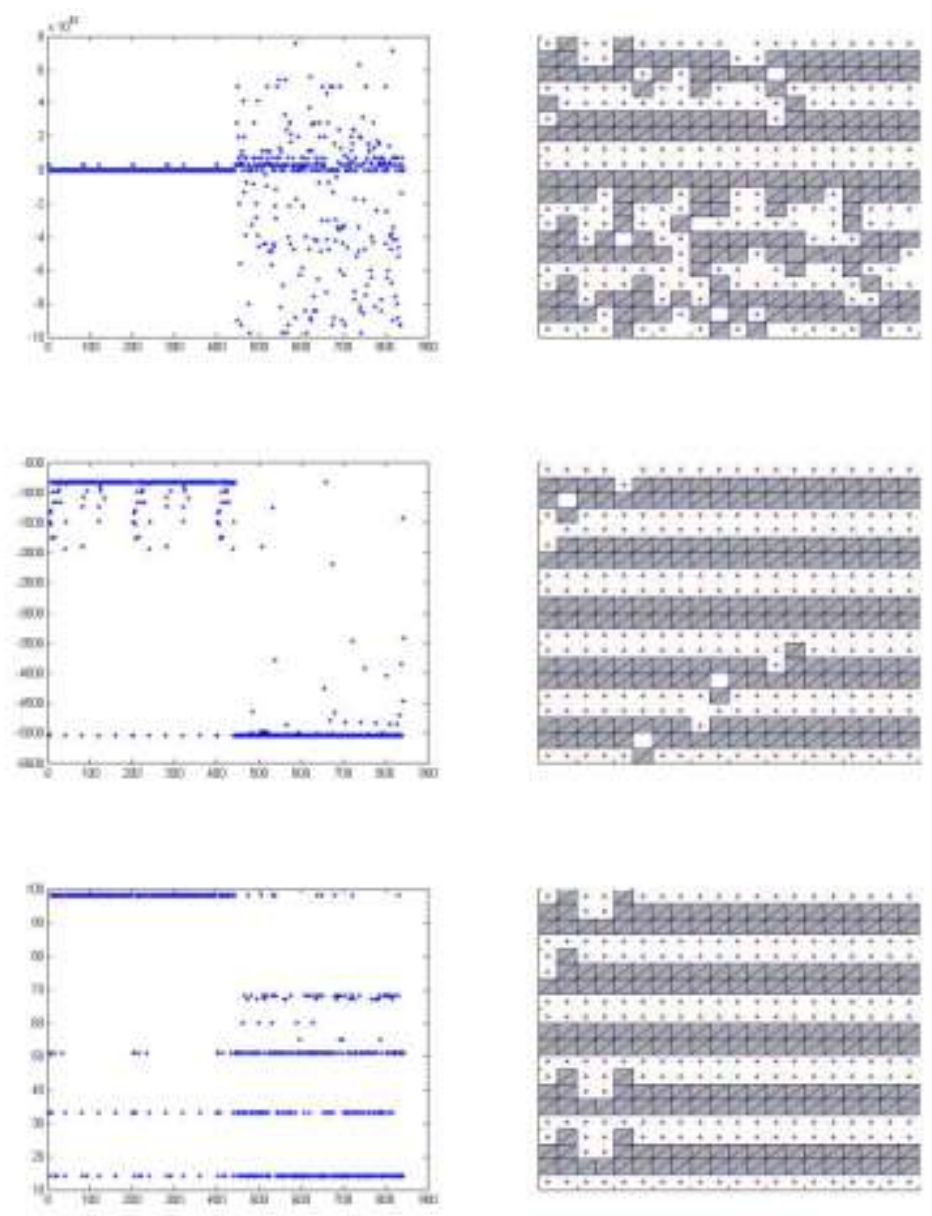

Figure 13: Three completely different algorithms (two double-layer neural networks and one Kohonen network [46]) result in different feature spaces (left), but make similar evaluations and similar constructions.

\section{CONCLUSION}

The idea of a style in any discipline is a fluid concept that is always subject to change, and therefore suited to a flexible representation. What is suggested here is that it can nevertheless be accurately represented and emulated. This work has presented an algorithmic method for both deriving a stylistic definition automatically from examples, and using it to generate new designs. Architectural examples were used, and were investigated primarily in terms of their spatial features, but it is intended as a general model in that other forms of input and classification algorithms may be used. Likewise, axial analysis and the 
aggregation model are not essential to the method, but the principles of feature space reduction and archetype should apply to a variety of analysis and synthesis techniques.

The concept of the archetype proposed is of a defined ideal and of a space in which to measure example designs. It contains only the features most relevant to define that style, but they are not counted as symbolic wholes. Instead one can measure an example's similarity in degrees, on an objective and continuous scale. This results in a definition of style that is flexible, can evolve, and is based on examples as per the criteria in section 2.3 .

A few final words on the relevance to human perception of style are appropriate in conclusion. Style is a social phenomenon, in that it requires a context of designers producing works both within and outside any given style. Prototypes or real buildings are necessary embodiments of a style, and these are the medium of its communication. While the details of this mechanism are beyond the scope of this paper (see also [41]), this model is based on the notion that the style is defined by the particular corpus of work. This corpus, in turn, may be explicitly selected and dogmatically transmitted as in the experiments of section 5 , or it may be obvious to any human observer or unsupervised classifier algorithm as in section 4 , where a wide margin between clusters is clear. Both situations certainly occur in human understanding of style.

The initial space of data input is objective, as all features are of equal significance within it, but the feature space associated with the style only represents those that are dominant and essential to that style. As such, archetypes do not correspond to individual buildings in all their detail, but only in these essential features. Furthermore, the fact that the feature space is derived from examples implies that even these essential features may change from style to style. The spaces of two styles may overlap, or the borders may be drawn tighter or wider, just as the history of architecture reveals both determined distinctions and gradual evolutions.

The definition of style provided by the archetype is analytical rather than generative, but there is still an obvious role for generative systems to play. The aggregation model in section 4 was chosen for its simplicity and common origin with the analysis in the previous section, but shape grammars and other generative rules could be applied - a likely avenue for future exploration. Their role in this regard however, is as a framework for exploration of many styles rather than a definition of one. 
Creative design is ultimately not a matter of rule following, but of judgement, and the model presented here proposes the flexibility this implies may extend to the definition of styles themselves.

\section{Acknowledgements}

I would like to thank professors Alan Penn and Philip Steadman for space syntax related advice, and for introducing some of the background examples presented in this paper. This research has been supported in part by the Engineering and Physical Sciences Research Council, UK and by Foster and Partners.

\section{References}

1. Chomsky N: 1957, Syntactic Structures, Mouton, The Hague.

2. Hersey, GL and Freedman, R: 1992, Possible Palladian villas: (plus a few instructively impossible ones). The MIT Press, Cambridge MA.

3. Koning H and Eizenberg J: 1981, The language of the prairie: Frank Lloyd Wright's prairie houses, Environment and Planning B: Planning and Design, vol. 8 pp. 295-323

4. Stiny G and Mitchell WJ: 1978, The Palladian grammar. Environment and Planning B: Planning and Design, vol. 5 pp. 5-18.

5. Duda, RO, Hart, PE and Stork DG: 2001, Pattern classification. John Wiley \& Sons, NY.

6. Gibson JJ: 1979, The Ecological Approach to Visual Perception. Houghton Mifflin, Boston.

7. Gombrich E H: 1960, Art and Illusion. Phaidon, London.

8. Stiny G: 1976, Two exercises in formal composition. Environment and Planning B: Planning and Design, vol. 3 pp. 187-210.

9. Stiny G: 1980, Introduction to shape and shape grammars. Environment and Planning B: Planning and Design, vol. 7 pp. 343-351. 
10. Prats M, Earl C, Garner S and Jowers I: 2006, Exploring style through generative shape description, AIEDAM Journal, Cambridge University Press, 20(3).

11. Knight TW: 1998, Shape Grammars, Environment and Planning B: Planning and Design, Anniversary Issue, 1998, pp. 86-91.

12. Chan, CS: 1994, Operational definitions of style, Environment and Planning B: Planning and Design, vol. 21 pp. 223-246.

13. Alexander, C, Ishikawa, S, Silverstein, M, Jacobsen, M, Fiksdahl-King, I and Angel, S: 1977, A Pattern Language, Oxford University Press, New York.

14. Koile, K: 1997, Design conversations with your computer: evaluating experiential qualities of physical form, CAAD futures 1997, pp. 203-218.

15. Koile, K: 2004, An intelligent assistant for conceptual design, in Gero, JS (ed), Design Computing and Cognition '04. Kluwer, Dordrecht. pp. 3-22.

16. Jupp J, and Gero, JS: 2003, Towards computational analysis of style in architectural design. in S Argamon (ed), IJCAIO3 Workshop on Computational Approaches to Style Analysis and Synthesis, IJCAI, Acapulco, pp 1-10

17. Gero, JS and Kazakov, V: 2001, Entropic-based Similarity and Complexity Measures of 2D Architectural Drawings, in Gero, JS, Tversky, B and Purcell, T (eds), Visual and Spatial Reasoning in Design II, Key Centre of Design Computing and Cognition, Sydney.

18. Jupp, J and Gero, JS: 2004, A Characterisation of 2D Architectural Style, Journal of the American Society of Information Science.

19. Goodman N: 1975, The Status of Style. Critical Inquiry, vol. 1. Reprinted in Goodman N: 1978, Ways of Worldmaking. Hackett Publishing Company Inc. Indianapolis.

20. Vitruvius: 2001, Ten Books on Architecture. Cambridge University Press. 
21. Arthur WB, Durlauf S and Lane DA: 1997, Process and Emergence in the Economy, in Arthur WB, Durlauf S and Lane DA (eds.) The Economy as an Evolving Complex System II. AddisonWesley, Reading, Mass., 1997

22. Hillier B and Hanson J: 1984, The Social Logic of Space. Cambridge University Press.

23. Duarte JP, Ducla-Soares G, Caldas JG and Rocha J: 2006, An Urban Grammar for the Medina of Marrakech: Towards a Tool for Urban Design in Islamic Contexts. In Gero JS (ed.) Design Computing and Cognition '06, Springer, pp. 483-502.

24. Cope D: 2001, Virtual Music: Computer Synthesis of Musical Style. MIT Press, Cambridge, MA.

25. Tillmann, B, Abdi, H and Dowling, WJ: 2004, Musical style perception by a linear auto-associator model and human listeners, Proceedings of the $8^{\text {th }}$ International Conference on Mucic Perception \& Cognition, Evanston, IL.

26. Turk, M and Pentland, A: 1991, Eigenfaces for Recognition, Journal of Cognitive Neuroscience, 3(1) pp. 71-86.

27. Burgess C and Lund K: 1997, Modelling parsing constraints with high-dimensional semantic space. Language and Cognitive Processes, 12, pp. 1-34.

28. Durrant-Whyte H: 2004, Autonomous navigation in unstructured environments, Proceedings of the 8th International Conference on Control, Automation, Robotics and Vision.

29. Turner A, Doxa M, O'Sullivan D, and Penn A: 2001, From isovists to visibility graphs: a methodology for the analysis of architectural space. Environment and Planning B: Planning and Design, 28(1) 103-121.

30. Hillier B, Hanson J, Peponis J, Hudson J and Burdett R: 1983, Space Syntax, Architects Journal, 178(48), pp 67-75. 
31. Desyllas, J and Duxbury E: 2001, Axial Maps and Visibility Graph Analysis, Proceedings, $3^{\text {rd }}$ International Space Syntax Symposium, Georgia Institute of Technology Atlanta.

32. Peponis J, Hadjinikolaov E, Livieratos C and Fatouros DA: 1989, The spatial core of urban culture, Ekistics 56(334/335), pp. 43-55.

33. Hillier B, Penn A, Hanson J, Grajewski T and Xu J: 1993, Natural movement, Environment and Planning B: Planning and Design, vol. 20 pp. 29-66.

34. Spiliopoulou G and Penn A: 1999, Organisations as Multi-Layered Networks, Proceedings, $2^{\text {nd }}$ Intl. Space Syntax Symposium, pp. 1-24.

35. Desyllas, J: 2000, The Relationship between Urban Street Configuration and Office Rent Patterns in Berlin, PhD thesis, Bartlett School of Graduate Studies, UCL, London.

36. Hillier B and Shu S: 2001, Crime and urban layout: The need for evidence, in Ballintyne S, Pease, K and McLaren V: Secure Foundations: Key Issues in Crime Prevention and Community Safety. IPPR, London.

37. Dalton R C, and Kirsan C: 2005, Small Graph Matching and Building Genotypes. Environment and Planning B: Planning and Design (forthcoming).

38. Robles-Kelly A, and Hancock E R: 2003, Edit Distance From Graph Spectra. Proceedings of the ninth IEEE International Conference on Computer Vision (ICCV 2003)

39. Weston, R: 2004, Key buildings of the twentieth century: plans, sections, elevations. W W Norton \& Co. Inc., New York.

40. Turner, A: 2005, An Algorithmic Definition of the Axial Map, Environment and Planning B: Planning and Design, 32(3) 425-444. 
41. Hanna S: 2005, Where Creativity Comes From: the social spaces of embodied minds, in Gero JS and Maher ML (eds.) Computational and Cognitive Models of Creative Design, Key Centre of Design Computing and Cognition, Sydney.

42. Tang H-H and Gero J: 2001, Sketches as affordances of meanings in the design process. In J. S. Gero, B. Tversky and T. Purcell (eds), 2001, Visual and Spatial Reasoning in Design II, University of Sydney, Australia, pp. 271-282.

43. Brockman JB and Director SW: 1991, The Hercules Task Management System, in Procedings of the International Conference on Computer-Aided design.

44. Vapnik V: 1995, The Nature of Statistical Learning Theory. Springer-Verlag, New York.

45. Rosenblatt F: 1958, The Perceptron: A Probabilistic Model for Information Storage and Organization in the Brain, Psychological Review, 65(6) pp. 386-408.

46. Kohonen T: 1982, Self-organized formation of topologically correct feature maps, Biological Cybernetics, 43(1) pp. 59-69. 